\title{
Protective effect of SIRT3 on acute lung injury by increasing manganese superoxide dismutase-mediated antioxidation
}

\author{
YONG GANG TIAN and JIAN ZHANG \\ Department of Critical Care Medicine, Shengli Oilfield Central Hospital, Dongying, Shandong 257034, P.R. China
}

Received November 23, 2015; Accepted December 19, 2016

DOI: $10.3892 / \mathrm{mmr} .2018 .8469$

\begin{abstract}
Prolonged exposure to hyperoxia results in acute lung injury (ALI). Pulmonary damage caused by oxygen toxicity occurs due to the generation of reactive oxygen species and subsequent formation of more potent oxidants. The present study demonstrated that sirtuin 3 (SIRT3) may attenuate hyperoxia-induced ALI due to its potential antioxidative effect. In the present study, a hyperoxia-induced acute lung injury mouse model, reverse transcription-quantitative polymerase chain reaction, western blotting, retroviral mediated gene over-expression and knockdown assays revealed that the expression of SIRT3 in the lung tissue of mice with hyperoxia-induced ALI was decreased and overexpression of SIRT3 may significantly reduce hyperoxia-induced ALI, as reflected by decreases in protein concentration, infiltrated neutrophils in bronchoalveolar lavage (BAL) fluid and wet/dry ratio of lung tissues. Furthermore, overexpression of SIRT3 increased the protein levels and enzymatic activity of manganese superoxide dismutase (MnSOD), and inhibited oxidative stress in the lungs of ALI mice. Additionally, the current study demonstrated that SIRT3 promoted the expression of MnSOD, and this regulation was crucial for the protective effect of SIRT3 on hyperoxia-induced ALI. In summary, the results of the current study indicated that SIRT3 overexpression may effectively ameliorate hyperoxia-induced ALI in mice, which indicates a potential application for SIRT3-based gene therapy to treat clinical adult respiratory distress syndrome.
\end{abstract}

\section{Introduction}

Supplemental oxygen is a common lifesaving strategy used in neonatal intensive care units (1). Oxygen therapy utilizing supraphysiological concentrations of oxygen (hyperoxia) is routinely administered during mechanical ventilation for the

Correspondence to: Dr Jian Zhang, Department of Critical Care Medicine, Shengli Oilfield Central Hospital, 31 Ji Nan Road, Dongying, Shandong 257034, P.R. China

E-mail: zhangjiandoc@126.com

Key words: sirtuin 3, hyperoxia-induced acute lung injury, superoxide dismutase, antioxidation management of severe respiratory distress (2-4). However, oxygen therapy can also cause oxygen toxicity, including acute lung injury (ALI) $(5,6)$. Additionally, exposure to high concentrations of oxygen may induce diffuse pulmonary injuries, vascular leakage, excessive inflammation and pulmonary edema $(7,8)$. Hyperoxia-induced damage to lung tissues is attributed to the generation of reactive oxygen species (ROS) and the subsequent formation of more potent oxidants (9-11). Therefore, excessive levels of ROS and the resultant oxidative damage have an important role during the process of hyperoxia-induced ALI $(12,13)$. As the understanding of the mechanism of hyperoxia-induced ALI is incomplete, effective therapies have not yet been developed.

Sirtuins are protein deacetylases that hydrolyze one oxidized nicotinamide adenine dinucleotide (NAD+) for each lysine residue that they deacetylate. Thus, their activity is associated with cellular energy levels $(14,15)$. Sirtuins were initially investigated as mediators of the increased lifespan that is associated with calorie restriction in yeast $(16,17)$; however, recent studies indicate that they are involved in a variety of functions, including genomic stability, tumorigenesis, inflammation and metabolic diseases (18). In mammals, sirtuins are comprised of seven proteins (SIRT1-7), which have different subcellular localizations. SIRT1 and 2 are present in the nucleus and cytoplasm, SIRT3-5 are located in the mitochondria, and SIRT6 and 7 are located in the nucleus (19). The majority of studies have focused on SIRT1 and 2, and the investigation of other SIRTs has been less extensive.

SIRT3 is an important mitochondrial protein. It controls various aspects of mitochondrial function by deacetylating various mitochondrial matrix proteins, including antioxidant effectors and proteins involved in the electron transport chain, therefore acting as a tumor suppressor by limiting the production of ROS. SIRT3 is important for mitochondrial function, by limiting oxidative stress and reducing ROS production, which results in a decrease in mitochondrial membrane potential (20). A previous study demonstrated that SIRT3 enhanced the expression of certain antioxidant proteins, including mitochondrial superoxide dismutase (SOD) (21).

SODs are enzymes that alternately catalyze the dismutation, or partitioning, of the toxic superoxide radical into either ordinary molecular oxygen or hydrogen peroxide. Superoxide is produced as a by-product of oxygen metabolism and causes various types of cell or tissue damage. Thus, SOD is a major antioxidant defense in almost all living cells that are exposed 
to oxygen. The following four isoforms of SOD are present in mammalian cells: Manganese superoxide dismutase (MnSOD), copper-zinc superoxide dismutase (CuZnSOD), extracellular SOD (ecSOD) and glutathione peroxidase 1 (GPx1). Studies using exogenous MnSOD or genetically altered mice overexpressing MnSOD demonstrated that MnSOD inactivates mitochondrial ROS and ameliorates hyperoxia-induced ALI (22). Additionally, CuZnSOD, which is evenly distributed intracellularly, is present in the nucleus and lysosomes. A previous study demonstrated that CuZnSOD was expressed in the alveolar epithelium, mesenchymal cells, fibroblasts and vascular endothelial cells of rat lungs (23). By contrast, ecSOD is primarily located in the extracellular matrix and expressed in the bronchial epithelium, alveolar epithelium and alveolar macrophages (24).

Based on the evidence discussed, we hypothesized that SIRT3 may have pharmacological effects on hyperoxia-induced ALI and that the potential antioxidative mechanism may be caused by regulating the expression of SOD in mice. Therefore, the aim of the current study was to investigate whether SIRT3 was able to inhibit the oxidative damage observed during hyperoxia-induced ALI by increasing the expression of SOD.

\section{Materials and methods}

Animals. Eighty adult pathogen-free female C57BL/6 mice (6-8-weeks-old, weight $20 \pm 5 \mathrm{~g}$ ) were provided by the SLRC Laboratory (Shanghai, China). Animals were raised under standard conditions and were provided with water and food ad ibitium, with a $12 \mathrm{~h}$ day/night cycle, and were acclimatized to their environment for at least one week prior to the initiation of the experiments. The study was approved the Ethics Committee of Shengli Oilfield Central Hosptal. Animal care and handling were performed in accordance with the National Institutes of Health guidelines.

Retrovirus preparation and infection. Retroviral vectors containing either the SIRT3 gene or SIRT3 small interfering RNA (siRNA) were constructed according to the sequence information from NCBI (NCBI reference sequence: NM_022433.2). RNAi design software (Invitrogen; Thermo Fisher Scientific, Inc., Waltham, MA, USA) was utilized for the sequence design. The sequence for the SIRT3 siRNA was: 5'-GCAAUAGAUUUAAUGACAG-3'. Retrovirus vectors (SIRT3 overexpression and SIRT3 siRNA) were transfected into 293 cells using calcium phosphate precipitation (25). After $24 \mathrm{~h}$, the medium was changed to DMEM containing $10 \%$ FBS. The cells were cultured for another $24 \mathrm{~h}$, and then the supernatant containing the lentivirus was harvested.

Animal model of hyperoxia-induced ALI. Mice were randomly divided into the following four groups: Control $\left(\mathrm{n}=20\right.$; mice subjected to normal air containing $21 \% \mathrm{O}_{2}$; Ctr group); hyperoxia-induced ALI model group ( $n=20$; mice subjected to $90 \% \mathrm{O}_{2}$; Hyper group); vector-carrying retrovirus (vector-RV)-treated ALI group $(n=20$; mice subjected to $90 \% \mathrm{O}_{2}$ and received lentivirus containing only vector via tail vein injection; Hyper + vector group) and SIRT3-overexpressing retrovirus (SIRT3-RV)-treated ALI group $\left(\mathrm{n}=20\right.$; mice subjected to $90 \% \mathrm{O}_{2}$ and received lentivirus containing SIRT3 via tail vein injection; Hyper + SIRT3 group). The mice were treated with vector or SIRT3-RV 3 days after exposure to $90 \% \mathrm{O}_{2}$.

To induce hyperoxia-induced ALI, mice were allowed to roam free under normobaric pressures in chambers under $90 \% \mathrm{O}_{2}$ or normal air containing $21 \% \mathrm{O}_{2} \cdot \mathrm{O}_{2}$ mixtures or normal air were delivered through the chamber at $3 \mathrm{l} / \mathrm{min}$ and allowed to vent through a distal ventilation port to maintain normobaric pressures. After 6 days of exposure, mice were terminally anesthetized with ketamine intraperitoneally (80 mg/kg body weight). Subsequently, under sterile conditions, thoraxes were opened, and blood was sampled by cardiac puncture. Simultaneously, three bronchoalveolar lavage (BAL) procedures were performed, each with $1 \mathrm{ml}$ of normal saline. Fluid and blood were centrifuged $(2,000 \mathrm{x}$ g, for $10 \mathrm{~min}$ at $4^{\circ} \mathrm{C}$ ) and the supernatant and plasma were stored for further processing. Survival of mice following ALI induction and group-specific treatments were assessed, and the cumulative survival curve was produced using the Kaplan-Meier method.

Bronchoalveolar lavage fluid (BALF) collection and determination of cytokine and protein concentration. At the end of the procedure, the right lungs were ligated at the right main bronchus and the BALF was collected from the left lung through a tracheal cannula with $5 \mathrm{ml}$ of sterile PBS. The collected BALF was centrifuged at $300 \mathrm{x} \mathrm{g}$ for $10 \mathrm{~min}$ at $4^{\circ} \mathrm{C}$, and the supernatants were stored at $-70^{\circ} \mathrm{C}$. The protein concentration in the BALF supernatants was determined by the Bradford method (Bio-Rad Laboratories, Inc., Hercules, CA, USA) with bovine serum albumin (Sigma-Aldrich; Merck Millipore, Darmstadt, Germany) used as a reference standard.

BAL cell counts. The trachea was exposed via a midline incision and the lungs were gently lavaged via a tracheal mannula with three aliquots of $1 \mathrm{ml}$ PBS (0.5 M, pH 7.4). The volume of the recovered lavage fluid was recorded, and cell counts were determined using a hemocytometer. Differential counts were performed on cells stained with Wright-Giemsa stain as previously described (26).

Histopathological grading of hyperoxia-induced $A L I$. Histopathological evaluation was performed on paraffin-embedded tissues as described previously (27). Prior to removal, the lungs were rinsed with PBS and then instilled with $1 \mathrm{ml}$ of buffered formalin through an angiocatheter inserted in the trachea. The lungs were then paraffin embedded, and these paraffin blocks were sliced into $5 \mu \mathrm{m}$ sections. Five random $5 \mu \mathrm{m}$ thick paraffin-embedded tissue sections from five different mice lungs taken at day 6 of ALI treatment were stained with hematoxylin and eosin (H\&E). The histopathology analysis was performed using a conventional light microscope (Olympus BX51; Olympus Corporation, Tokyo, Japan) and images were captured using a Nikon DXM1200C digital camera (Nikon Corporation, Tokyo, Japan).

To assess the severity of the lung injury, a semi-quantitative histological index was used. Five sections were randomly selected from each group of mice, and five fields were examined per section. The lung histopathological changes were scored on a scale of 0-5 according to the degree of congestion, lung edema, infiltration of inflammatory cells and hemorrhage 
in lung tissues (28). A score of 0 indicated no injury in lung tissues, 1 indicated modest injury, 2 indicated intermediate injury, 3 indicated widespread injury and 4 indicated severe injury. The overall score of hyperoxia-induced ALI was generated based on the summation of all scores, and the mean + standard error of the mean (SEM) of the scores were calculated for the lungs of the normal air controls.

Cell culture. A549 cells, a tumor cell line derived from a human lung carcinoma with properties of type II alveolar epithelial cells, and 293 cells were purchased from Cell Resource Center of Chinese Academy of Sciences (Shanghai, China). The cells were cultured in Dulbecco's modified Eagle's medium (Invitrogen; Thermo Fisher Scientific, Inc.) containing 10\% heat-inactivated fetal calf serum (Gibco/Invitrogen; Thermo Fisher Scientific, Inc.), and cultured in a $5 \% \mathrm{CO}_{2}-95 \%$ air chamber at $37^{\circ} \mathrm{C}$. For the delivery of Ritrovirus into A549 cells, the cells were resuspended with virus solution, and then the plates were centrifuged at $1,500 \mathrm{x}$ g for $120 \mathrm{~min}$ at $4^{\circ} \mathrm{C}$.

Western blotting. The levels of SIRT3 and MnSOD protein in lung tissue were measured using western blot analysis. The lung tissues of treated and control mice, and differentially treated cells, were homogenized, washed with PBS, incubated in lysis buffer for $30 \mathrm{~min}$ at $4^{\circ} \mathrm{C}$, and a mixture of protease inhibitors was added (Sigma-Aldrich; Merck Millipore) to obtain extracts of tissue or cell proteins. The protein concentration in the supernatant was determined using the Bradford assay. Briefly, total protein $(50 \mu \mathrm{g})$ was loaded into each lane. The proteins were transferred onto polyvinylidene fluoride membranes following $10 \%$ SDS-PAGE, and the membranes were blocked with $5 \%$ non-fat milk for $1 \mathrm{~h}$ at room temperature. Subsequently, membranes were incubated with primary antibodies overnight at $4^{\circ} \mathrm{C}$, and the membranes were washed, incubated with secondary antibodies for $1 \mathrm{~h}$ at room temperature, and visualized by enhanced chemiluminescence reagent (Thermo Fisher Scientific, Inc.). The following antibodies were used: Rabbit anti-mouse SIRT3 polyclonal antibody (cat. no. ab86671; Abcam, Cambridge, UK); rabbit anti-mouse MnSOD polyclonal antibody (cat. no. PA1-125; Thermo Fisher Scientific, Inc.) and rabbit anti-mouse $\beta$-actin antibody (cat. no. ab189073; Abcam). Horseradish peroxidase-conjugated goat anti-rabbit IgG (cat. no. ab6721; Abcam) was used as secondary antibody. The dilutions for all antibodies were 1:1,000. ImageJ version 1.46r software (National Institutes of Health, Bethesda, MD, USA) was used for densitometric analysis. The experiment was repeated three times.

RNA isolation and reverse transcription-quantitative polymerase chain reaction $(R T-q P C R)$. RNA from treated lung tissues and cells were isolated using TRIzol ${ }^{\circledR}$ reagent (Invitrogen; Thermo Fisher Scientific, Inc.). Total RNA $(0.4 \mu \mathrm{g})$ was then treated with RNase-free Dnase (1 U/sample, Sigma-Aldrich; Merck Millipore), and cDNA was generated using random hexamer primers provided in the RevertAid First-Strand cDNA synthesis kit (Applied Biosystems; Thermo Fisher Scientific, Inc., Cat no. K1621). The products of RT reaction were diluted and used as templates in subsequent qPCR or stored at $-20^{\circ} \mathrm{C}$. qPCR analysis was performed using a sequence detection system (7900HT Fast Real-Time PCR system; Applied Biosystems; Thermo Fisher Scientific, Inc.). Specifically, diluted cDNA sample was amplified using the SYBR Green PCR kit (Invitrogen; Thermo Fisher Scientific, Inc.). Thermal cycling was initiated with an initial denaturation step of $5 \mathrm{~min}$ at $95^{\circ} \mathrm{C}$, followed by 40 cycles of $95^{\circ} \mathrm{C}$ for $10 \mathrm{sec}$ and $60^{\circ} \mathrm{C}$ for $30 \mathrm{sec}$. The number of replicates per sample was 40 and the $2^{-\Delta \Delta \mathrm{Cq}}$ method was used to analyze the results (29). The following mouse-specific primer pairs were used: $\beta$-actin, 5'-GGCCAGGTCATCACTATTG-3' (forward) and 5'-GAGGTCTTTACGGATGTCAAC-3' (reverse); CuZnSOD, 5'-GACAAACCTGAGCCCTAAG-3' (forward) and 5'-CGACCTTGCTCCTTATTG-3' (reverse); MnSOD, 5'-ATGTCTGTGGGAGTCCAA-3' (forward) and 5'-TGA AGGTAGTAAGCGTGCTC-3' (reverse); ecSOD, 5'-ATTTCAGTCTGGAGGGCT-3' (forward), 5'-CACGAA GTTGCCAAAGTC-3' (reverse); GPx1, 5'-GACTACACC GAGATGAACGAT-3' (forward) and 5'-CACTTCGCACTT CTCAAACA-3' (reverse); SIRT3, 5'-CATCGACGGGCTTGA GAGA-3' (forward) and 5'-GGTCCCGTGGGCTTCAAC-3' (reverse). The Primer Express 3.0 software (https://www. thermofisher.com/order/catalog/product/4363991) was used to design the qPCR primers.

Lung wet/dry (W/D) ratio. The mice (40 in total) were anesthetized using sodium pentobarbital (intraperitoneally, $40 \mathrm{mg} / \mathrm{kg}$ ) and sacrificed via exsanguination 6 days after ALI induction. Right lungs were removed and the wet weights were obtained. Subsequently, the lungs were dried at $80^{\circ} \mathrm{C}$ and weighed again 3 days after drying. The W/D ratio was calculated to assess tissue edema. The W/D ratio was calculated as follows: (wet weight-dry weight)/dry weight (30).

Measurement of oxidized/reduced glutathione (GSH) ratio. The ratio of reduced GSH and oxidized GSH (GSSG) was determined in lung tissue homogenates from treated ALI mice by reaction with 5,5'-dithiobis-2-nitrobenzoic acid, using the Glutathione Assay kit (Merck Millipore) according to the manufacturer's instructions.

Lipid peroxidation. The lung tissues were immediately flash frozen in liquid nitrogen at time of harvest and stored at $-80^{\circ} \mathrm{C}$ to prevent auto-oxidation. Lipid peroxidation, a well-defined mechanism of cellular damage, was assessed by measuring the level of 8-isoprostane, an indicator of oxidative stress; 8-isoprostane levels were determined using an 8-isoprostane ELISA kit (Cayman Chemical Company, Ann Arbor, MI, USA) according to the manufacturer's instructions.

Measurement of tissue SOD activity. The BIOXYTECH ${ }^{\circledR}$ SOD-525 assay kit (OXIS Health Products, Inc., Portland, OR, USA) was used according to the manufacturer's instructions to measure SOD activity. Tissue SOD activity was determined by spectrophotometric detection of formazan production at $550 \mathrm{~nm}$, as a result of inhibition of nitroblue tetrazolium reduction, with xanthine-xanthine oxidase used as a superoxide generator, as described previously (31).

Survival study in mice with hyperoxia-induced ALI. Mice were randomly divided into four groups ( $n=10$ per group) as mentioned above. The survival rates were recorded at the 
indicated time points (day 1, 3, 5, 7, 9,11, 13 and 15 after treatment).

Statistical analysis. Data were analyzed using SPSS 13.0 software (SPSS, Inc., Chicago, IL, USA) and expressed as the mean \pm SEM. Significant differences were assessed by one-way analysis of variance followed by Fisher's least significant difference test. $\mathrm{P}<0.05$ was considered to indicate a statistically significant difference.

\section{Results}

SIRT3 expression is reduced in hyperoxia-induced ALI. Previous studies have demonstrated that sirtuins are highly conserved class III $\mathrm{NAD}^{+}$-dependent deacetylases that target histone proteins $(23,32-35)$. As a member of the sirtuin family, SIRT3 is reported to target mitochondrial proteins for lysine deacetylation and to regulate cellular functions. Evidence indicates that SIRT3 may regulate the mitochondrial adaptive antioxidant response (36-38). Therefore, the present study induced ALI in mice by exposure to high concentrations of $\mathrm{O}_{2}$ in order to investigate the function of SIRT3 in hyperoxia-induced ALI. The expression of SIRT3 mRNA and protein in the lung tissues of hyperoxia-induced ALI mice and control mice were then measured by RT-qPCR and western blot analysis. Representative histological sections of lung tissues from control mice (Fig. 1A) and hyperoxia-induced ALI mice (Fig. 1B) are presented. It was observed that the lungs from hyperoxia-induced ALI mice exhibited inflammatory infiltrations, edema and thickening of the alveolar walls, which was not observed in the control mice (Fig. 1A and B). The lung injury index that represents the severity of lung injury was significantly increased in the ALI group compared with the control mice $(\mathrm{P}<0.05$; Fig. 1C). To assess the expression of SIRT3, the mRNA and protein levels of SIRT3 in different mice lung tissues were measured. Compared with the controls, SIRT3 mRNA (Fig. 1D) and protein (Fig. 1E and F) expression in the lungs of hyperoxia-induced ALI mice were significantly decreased $(\mathrm{P}<0.05)$. These results indicated that SIRT3 may have an important role in hyperoxia-induced ALI.

Retrovirus treatment enhances SIRT3 expression in lung tissue. To determine the effect of a SIRT3-containing retrovirus on the expression of SIRT3 in the lung tissues, hyperoxia-induced ALI mice were treated with SIRT3-RV and vector-RV (retrovirus containing a blank vector as a control) via tail vein injection 3 days after ALI induction and the expression of SIRT3 protein in the differentially treated lung tissues was measured 3 days after injection by western blot analysis. As presented in Fig. 2, SIRT3 was overexpressed in the lung of SIRT3-RV treated ALI mice (Hyper + SIRT3), compared with the control ALI group (Hyper; $\mathrm{P}<0.05)$ and the vector-RV-treated ALI group (Hyper + vector; $\mathrm{P}<0.05$; Fig. 2).

Enhanced expression of SIRT3 protects against hyperoxia-induced ALI in mice. To further investigate the function of SIRT3 in hyperoxia-induced ALI, mice were treated with SIRT3-RV via tail vein injection. The effect of SIRT3-RV on lung histopathology is presented in Fig. 3. Treatment with SIRT3-RV reduced visible lung damage caused by exposure to a high concentration of $\mathrm{O}_{2}$, compared with untreated ALI mice and vector-treated ALI mice (Fig. 3A-D). The comparison of lung injury scores between groups was consistent with these findings; the Hyper + SIRT3 group demonstrated a lower score than Hyper and Hyper + vector groups (Fig. 3E). Additionally, compared with Hyper and Hyper + vector groups, treatment with SIRT3-RV significantly reduced the concentration of protein in BALF $(\mathrm{P}<0.05$; Fig. $3 \mathrm{~F})$, the wet/dry ratio $(\mathrm{P}<0.05$; Fig. $3 \mathrm{G})$, and the number of infiltrated neutrophils $(\mathrm{P}<0.05$; Fig. $3 \mathrm{H}$ ) in the lung tissue.

SIRT3 overexpression inhibits oxidation and the level of 8-isoprostane in lung tissues. It is known that oxidative stress and lipid peroxidation are involved in hyperoxia-induced ALI. The ratio of reduced/oxidized GSH (GSH/GSSG) reflects the oxidative status of tissues. To further determine the effect of SIRT3 on the hyperoxia-induced oxidative damage of lung tissues, the GSH/GSSG ratio in treated ALI mice was measured in the current study. It was observed that, compared with the control group, there was significant reduction in the ratio of GSH/GSSG in lung tissues of hyperoxia-induced ALI mice (Hyper; $\mathrm{P}<0.05$; Fig. 4A). Additionally, the current study demonstrated that the overexpression of SIRT3 (Hyper + SIRT3) significantly inhibited the reduction of the GSH/GSSG ratio caused by high $\mathrm{O}_{2}$ exposure, compared with the untreated ALI group (Hyper) and vector-treated ALI group (Hyper + vector; $\mathrm{P}<0.05$; Fig. 4A). 8-Isoprostane is one of the most reliable biomarkers of lipid peroxidation and oxidative stress. Therefore, the present study also measured the level of 8 -isoprostane in lungs and demonstrated that treatment with SIRT3-RV significantly reduced the level of 8-isoprostane 3 days after treatment, compared with the untreated ALI group (Hyper) and vector-treated group (Hyper + vector; $\mathrm{P}<0.05$; Fig. 4B). These results indicated that SIRT3 may have a potential antioxidative effect in hyperoxia-induced ALI.

Overexpression of SIRT3 enhances the total enzyme activity of SOD in ALI mice. Animal and human studies have indicated that acute and chronic lung injury due to hyperoxia may be ameliorated by the administration of antioxidants, such as SOD (39-43). Therefore, the enzyme activity of SOD in the lung tissues of differentially treated ALI mice was determined using a photometric assay that measured the autoxidation of a tetracyclic catechol. The current study demonstrated that SOD enzyme activity was significantly decreased in hyperoxia-induced ALI mice (Hyper) after 6 days of $\mathrm{O}_{2}$ inhalation, compared with the control group $(\mathrm{P}<0.05$; Fig. 5). Overexpression of SIRT3 in the lungs of hyperoxia-induced ALI mice (Hyper + SIRT3) increased the level of SOD enzymatic activity to a level comparable to the healthy controls (Fig. 5).

SIRT3 enhances the expression of MnSOD but has no effect on the expression of other SODs. Increased production of ROS, including superoxide, hydroxyl radicals and hydrogen peroxide is generally considered essential for enhancing $\mathrm{O}_{2}$ toxicity (44-47). Hyperoxia-induced injury increases the intracellular production of ROS, which occurs via the mitochondria. Additionally, an increasing number of studies suggest that SIRT3 may regulate the expression of SOD (48-51). Therefore, 

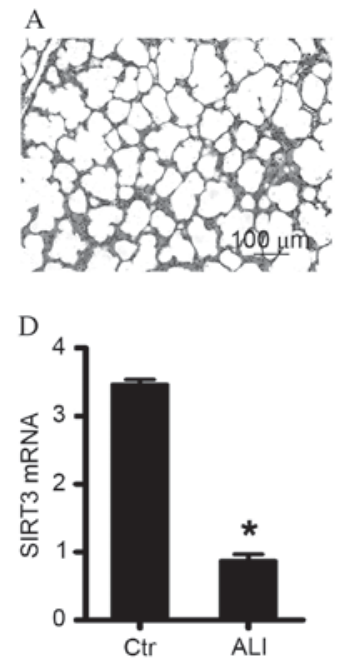

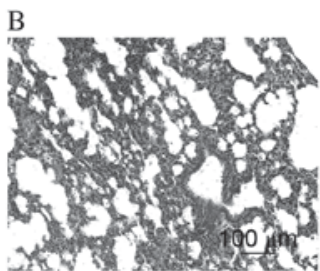

E

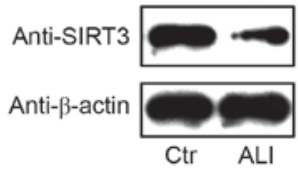

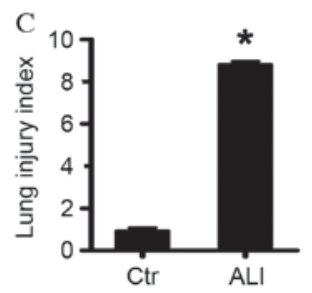

$\mathrm{F}$

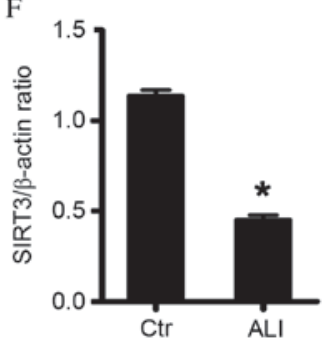

Figure 1. Hyperoxia-induced ALI and expression of SIRT3 in Ctr and ALI mice lung tissues. Hematoxylin and eosin-stained lung sections following 6 days of exposure to (A) normal air and (B) a high concentration of $\mathrm{O}_{2}$. (C) The lung injury index of Ctr and hyperoxia-induced ALI mice. (D) Relative level of SIRT3 mRNA in Ctr and hyperoxia-induced ALI mice. (E) Western blot analysis of SIRT3 protein levels. (F) Quantified western blot analysis of SIRT3 protein levels. Data are presented as the mean \pm standard error of the mean. ${ }^{*} \mathrm{P}<0.05$ vs. Ctr. Ctr, control; ALI, acute lung injury; SIRT3, sirtuin 3.
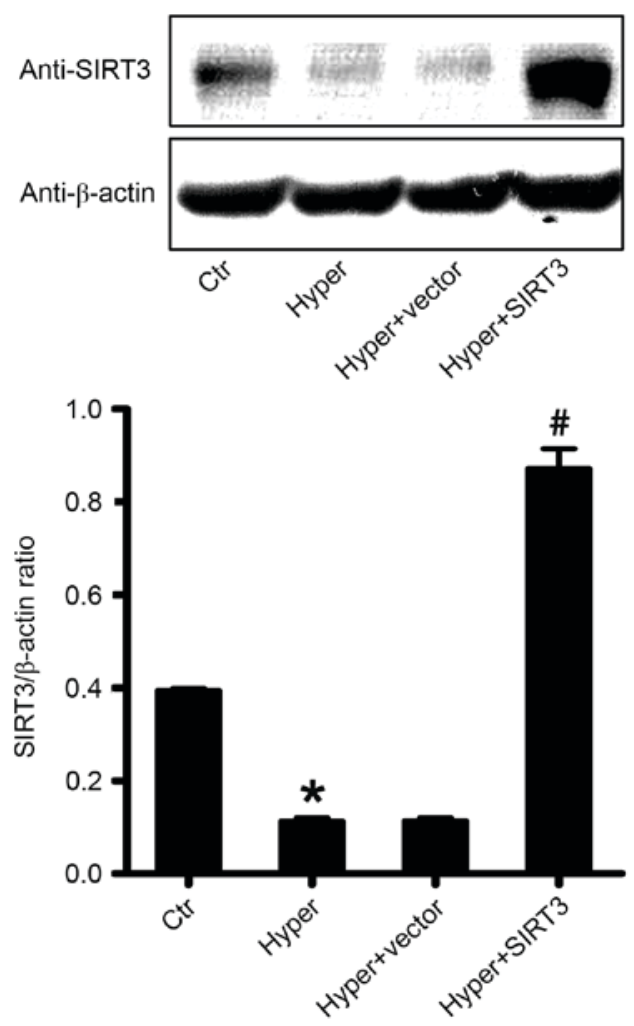

Figure 2. The expression of SIRT3 protein in lung tissues of ALI mice transduced with an RV containing the SIRT3 gene (SIRT3-RV) or vector (vector-RV), and control ALI mice. ${ }^{*} \mathrm{P}<0.05$ vs. $\mathrm{Ctr}$; ${ }^{\#} \mathrm{P}<0.05$ vs. hyperoxia-induced ALI group (Hyper) or vector-treated group (Hyper + vector) Data are presented as the mean \pm standard error of the mean. ALI, acute lung injury; RV, retrovirus; SIRT3, sirtuin 3; Ctr, control; Hyper, hyperoxia.

we hypothesized that SIRT3 may reduce hyperoxia-induced ALI by increasing the expression of SODs in vivo, as these enzymes scavenge ROS. The expression of MnSOD, CuZnSOD, ecSOD and GPx1 was measured by RT-qPCR in the current study. As presented in Fig. 6, compared with the untreated ALI group (Hyper) and vector-treated ALI group
(Hyper + vector), SIRT3 overexpression (Hyper + SIRT3) significantly increased the expression of MnSOD $(\mathrm{P}<0.05$; Fig. 6A), while the expression of the other SODs investigated, CuZnSOD (Fig. 6B), ecSOD (Fig. 6C) and GPx1 (Fig. 6D), was unchanged. These results indicated that SIRT3 overexpression may increase the expression of MnSOD in the lung tissue of hyperoxia-induced ALI mice and inhibit hyperoxia-induced ALI through the antioxidative effect of MnSOD in vivo.

Western blot analysis of MnSOD in mouse lung tissue. To further confirm the effect of SIRT3 on the expression of the MnSOD protein, the current study detected the protein levels of MnSOD in lung tissues using the western blot analysis. As presented in Fig. 7, MnSOD protein levels in the lungs of hyperoxia-induced ALI mice (Hyper) was significantly increased compared with control mice. Treatment with SIRT3-RV increased MnSOD protein levels induced by $\mathrm{O}_{2}$ exposure compared with the untreated ALI mice (Hyper) and the vector-treated ALI mice (Hyper + vector; P<0.05; Fig. 7).

SIRT3 enhances the expression of MnSOD in vitro. To further investigate the role SIRT3 may have in hyperoxia-induced ALI injury, the current study increased or decreased the expression of SIRT3 in human A549 cells using a retrovirus overexpressing SIRT3 or SIRT3 siRNA, respectively, followed by western blot analysis performed $24 \mathrm{~h}$ after cell transfection. As presented in Fig. 8A, compared with the control, overexpression of SIRT3 significantly increased the expression of MnSOD in vitro $(\mathrm{P}<0.05)$. The present study also demonstrated that the activity of SODs was significantly increased by overexpression of SIRT3, compared with the control $(\mathrm{P}<0.05$; Fig. 8B). These results are consistent with the observations from the in vivo studies.

SIRT3 overexpression improves survival following hyperoxia-induced $A L I$. To evaluate the long-term beneficial effect of SIRT3 on hyperoxia-induced ALI, the survival rate was compared between treated (Hyper + SIRT3) and control 

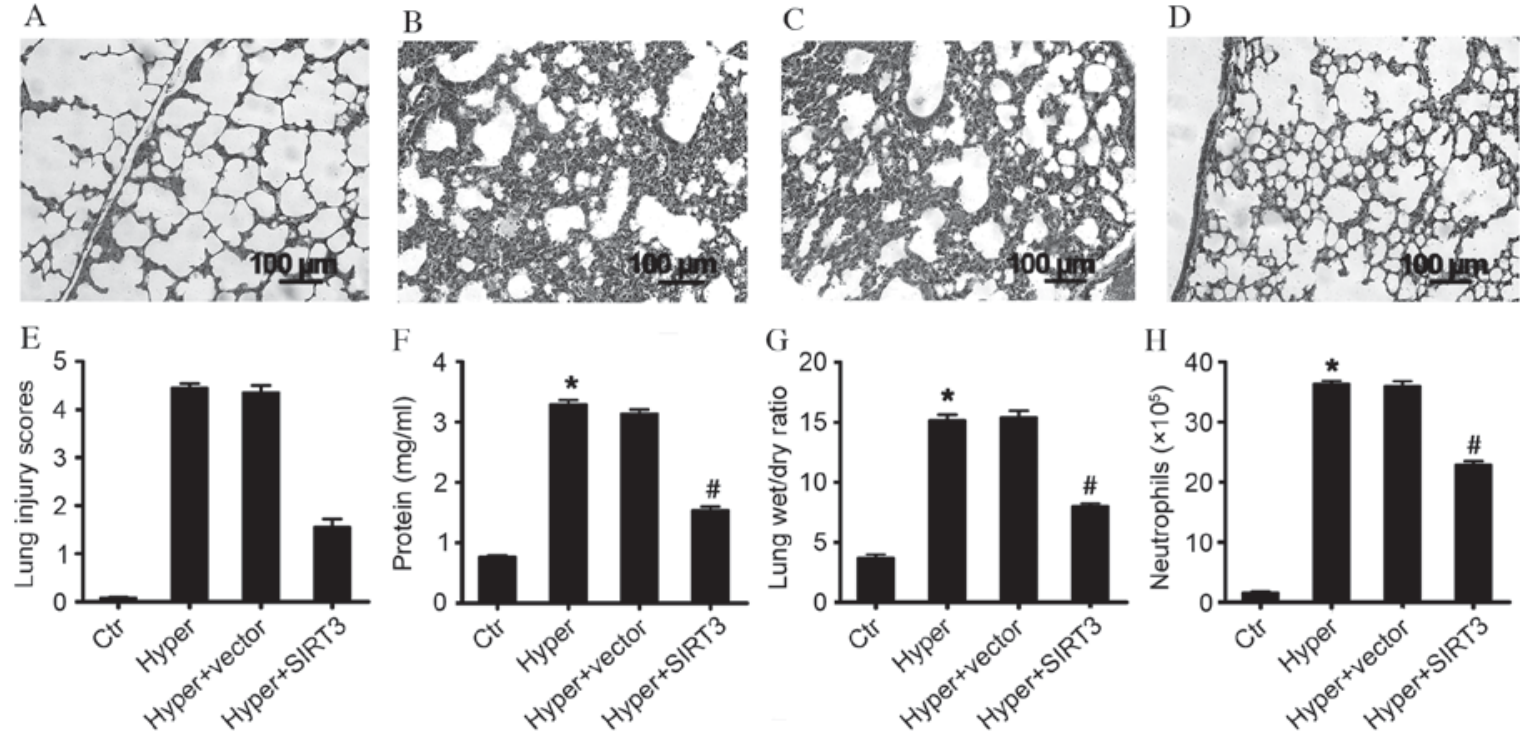

Figure 3. SIRT3 overexpression inhibits hyperoxia-induced ALI. Hematoxylin and eosin-stained lung sections following the different treatments in (A) normal air exposed controls, (B) high concentration $\mathrm{O}_{2}$ exposed group, (C) vector-treated group and (D) SIRT3 overexpression group. (E) The lung injury scores of differentially treated mice. (F) Protein concentration in BALF from differentially treated mice. (G) The wet/dry ratio of lung tissues from differentially treated mice. $(\mathrm{H})$ Number of infiltrated neutrophils in BALF from differentially treated mice. ${ }^{*} \mathrm{P}<0.05$ vs. $\mathrm{Ctr} ;{ }^{*} \mathrm{P}<0.05$ vs. hyperoxia-induced ALI group (Hyper) or vector-treated group (Hyper + vector). Data are presented as the mean \pm standard error of the mean. ALI, acute lung injury; BALF, bronchoalveolar lavage fluid; Ctr, control; Hyper, hyperoxia; SIRT3, sirtuin 3.
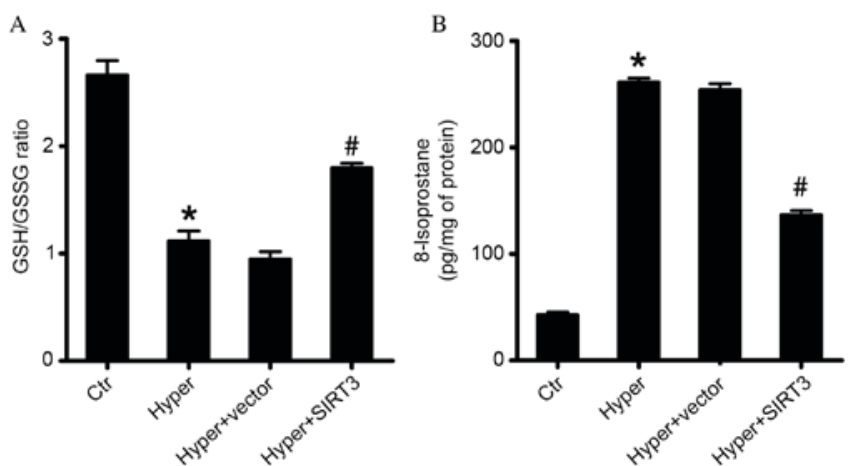

Figure 4. Ratio of GSH/GSSG and 8-isoprostane protein levels in lung tissues of mice from different treatment groups. (A) Ratios of GSH/GSSG in BALF of mice from different treatment groups. (B) 8-Isoprostane concentration in BALF of mice from different treatment groups. ${ }^{*} \mathrm{P}<0.05$ vs. $\mathrm{Ctr}$; ${ }^{\text {}} \mathrm{P}<0.05$ vs. hyperoxia-induced acute lung injury group (Hyper) or vector-treated group (Hyper + vector). Data are presented as the mean \pm standard error of the mean. BALF, bronchoalveolar lavage fluid; GSH, reduced glutathione; GSSG, oxidized glutathione; Ctr, control; Hyper, hyperoxia; SIRT3, sirtuin 3.

ALI mice (Hyper). The current study demonstrated that the survival rate was significantly improved in SIRT3-RV treated ALI mice compared with the untreated ALI mice $(\mathrm{P}<0.05)$ and vector-treated ALI mice ( $\mathrm{P}<0.05$; Fig. 9).

\section{Discussion}

Hyperoxia is a state of excess oxygen in tissues and organs. Oxygen toxicity occurs when the partial pressure of alveolar oxygen exceeds that which occurs when breathing normal air. With continuous exposure to supraphysiological concentrations of oxygen, a state of hyperoxia develops. Several studies have demonstrated that exposure of lung tissue to high concentration of oxygen causes oxidative stress and lung damage $(5,52-54)$.

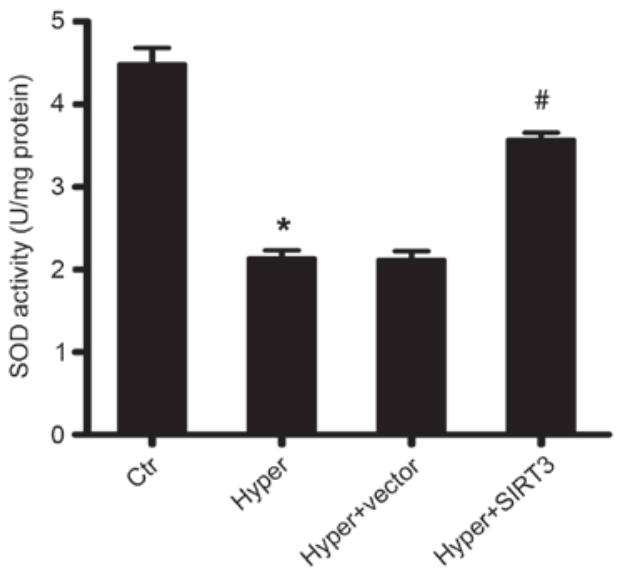

Figure 5. Total SOD enzyme activity in the lung tissues of differentially treated mice. ${ }^{*} \mathrm{P}<0.05$ vs. $\mathrm{Ctr}$; ${ }^{\#} \mathrm{P}<0.05$ vs. hyperoxia-induced acute lung injury group (Hyper) or vector-treated group (Hyper + vector). Data are presented as the mean \pm standard error of the mean. SOD, superoxide dismutase; $\mathrm{Ctr}$, control; Hyper, hyperoxia; SIRT3, sirtuin 3.
Lung oxidative stress results in an oxidant-antioxidant imbalance that can lead to various lung diseases, including adult respiratory distress syndrome. It is well established that oxidant production within the lung can lead to ALI (55-58). The present study demonstrated that SIRT3 overexpression reduced hyperoxia-induced ALI by increasing the expression of MnSOD and inhibiting the oxidative damage caused by hyperoxia induction.

Sirtuins are a family of highly conserved $\mathrm{NAD}^{+}$-dependent deacetylases that act as cellular sensors to detect energy availability and modulate metabolic processes. One sirtuin, SIRT3, is central to the control of metabolic processes, and is localized to the mitochondria, where the most damaging oxidants are generated $(33,59)$. Therefore, we hypothesized that SIRT3 
A

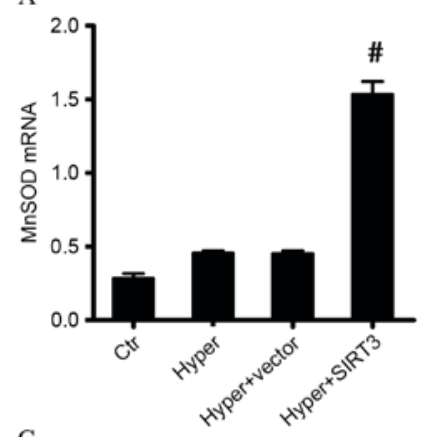

$\mathrm{C}$

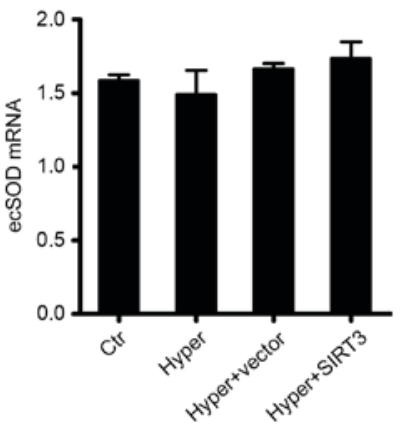

B



Figure 6. mRNA expression of SOD enzymes in the lung tissues of differentially treated mice. mRNA levels of (A) MnSOD, (B) CuZnSOD, (C) ecSOD and (D) GPx1. " $\mathrm{P}<0.05$ vs. hyperoxia-induced acute lung injury group (Hyper) or vector-treated group (Hyper + vector). Data are presented as the mean \pm standard error of the mean. SOD, superoxide dismutase; MnSOD, manganese SOD; Ctr, control; Hyper, hyperoxia; SIRT3, sirtuin 3; CuZnSOD, copper-zinc SOD; ecSOD, extracellular SOD; GPx1, glutathione peroxidase 1 .

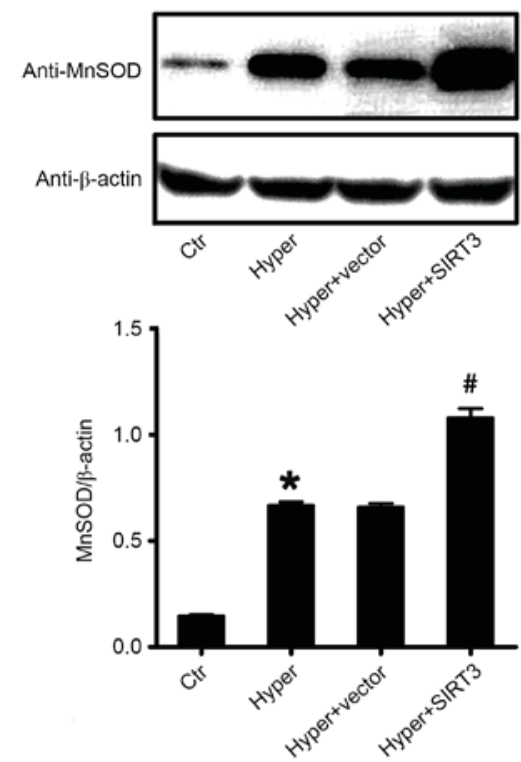

Figure 7. Protein levels of MnSOD in lung tissues of treated mice. MnSOD protein levels in each different treatment group. ${ }^{\text {"P }} \mathrm{P}<0.05$ vs. $\mathrm{Ctr}$; ${ }^{\text {}} \mathrm{P}<0.05$ vs. hyperoxia-induced acute lung injury group (Hyper) or vector-treated group (Hyper + vector). Data are presented as the mean \pm standard error of the mean. MnSOD, manganese superoxide dismutase; Ctr, control; Hyper, hyperoxia; SIRT3, sirtuin 3 .

may have an important role in hyperoxia-induced ALI. The results of this study demonstrated that lung injury was induced by exposure to a high concentration of $\mathrm{O}_{2}$, and reduced SIRT3

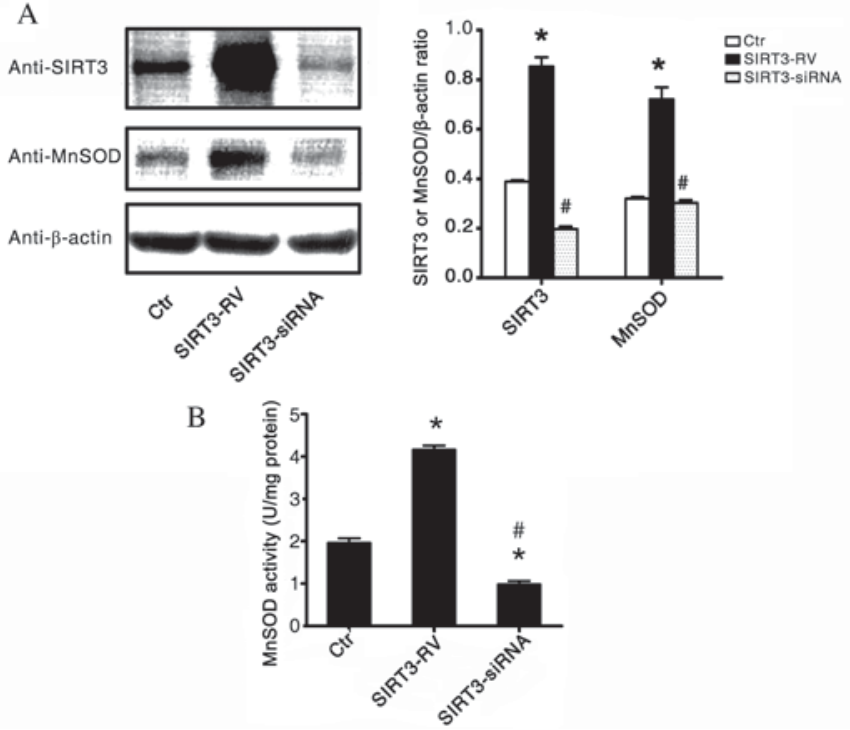

Figure 8. Effect of SIRT3 on the protein expression of MnSOD in vitro. (A) The protein expression of SIRT3 and MnSOD in differentially treated human A549 cells. (B) The activity of MnSOD in differentially treated human A5 49 cells. ${ }^{*} \mathrm{P}<0.05$ vs. $\mathrm{Ctr} ;{ }^{\#} \mathrm{P}<0.05$ vs. SIRT3-RV.) Data are presented as the mean \pm standard error of the mean. SIRT3, sirtuin 3; MnSOD, manganese superoxide dismutase; $\mathrm{Ctr}$, control; RV, retrovirus; siRNA, small interfering RNA.

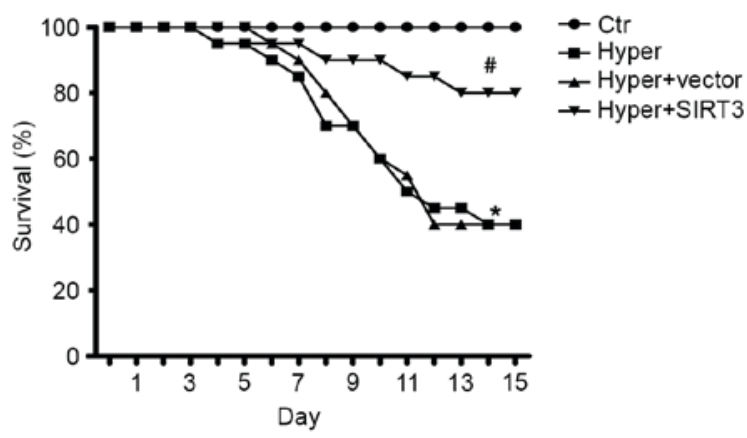

Figure 9. SIRT3 overexpression prolonged the survival of mice with hyperoxia-induced ALI. The Kaplan-Meier survival curves of mice $(n=10)$ with the indicated treatments were monitored. ${ }^{*} \mathrm{P}<0.05$ vs. $\mathrm{Ctr}$; ${ }^{\text {}} \mathrm{P}<0.05$ vs. hyperoxia-induced acute lung injury group (Hyper) or vector-treated group (Hyper + vector). Ctr, control; Hyper, hyperoxia; RV, retrovirus; SIRT3, sirtuin 3 .

levels was dependent upon hyperoxic exposure (Fig. 1). The data indicated that SIRT3 may have an important role in the process of hyperoxia-induced ALI.

To further investigate the effect of SIRT3 on hyperoxia-induced ALI, the current study treated ALI mice with a retroviral vector containing a SIRT3 gene (SIRT3-RV). The present study demonstrated that SIRT3 overexpression had a beneficial effect on hyperoxia-induced ALI (Fig. 3), including effects on the severity of lung damage (Fig. 3C), protein concentration in BALF (Fig. 3D), the lung wet/dry ratio (Fig. 3E) and the number of infiltrating neutrophils (Fig. 3F). Furthermore, the current study also demonstrated that SIRT3 overexpression increased the total enzyme activity of SODs (Fig. 5). These observations support the model that SIRT3 has a therapeutic effect on hyperoxia-induced ALI due to its antioxidative effect. Previous studies have reported 
that SIRT3 deacetylates MnSOD, thereby increasing MnSOD activity $(49,60)$. To explore the mechanism of the antioxidative effect of SIRT3, the mRNA expression of certain antioxidant enzymes that scavenge ROS, including MnSOD, CuZnSOD, ecSOD and GPx1 in the lung tissues of differentially treated mice were measured in the present study. The results demonstrated that SIRT3 overexpression increased the expression of MnSOD (Fig. 6A); however, it did not alter the mRNA levels of the other antioxidant enzymes, CuZnSOD (Fig. 6B), ecSOD (Fig. 6C) and GPx1 (Fig. 6D). These results were consistent with a previous report (61). To confirm the effect of SIRT3 on MnSOD, this effect was investigated in vitro. The current study inhibited or overexpressed SIRT3 in human A549 cells, and then detected the expression of SIRT3 and MnSOD proteins by western blot analysis. As presented in Fig. 8, the results demonstrated that SIRT3 significantly increased the expression of the MnSOD protein in vitro, compared with the control group. These results support the hypothesis that SIRT3 inhibits hyperoxia-induced ALI by increasing the expression of MnSOD, and thus inhibiting the oxidative damage caused by high concentration $\mathrm{O}_{2}$ exposure.

In conclusion, the results of the current study demonstrated that SIRT3 inhibited hyperoxia-induced ALI. As a mitochondrial protein, SIRT3 enhanced the expression of MnSOD and reduced the oxidative injury caused by hyperoxic exposure. SIRT3 may be useful as a target for the treatment of hyperoxia-induced ALI due to its potentially antioxidative effect.

\section{References}

1. Bhandari V: Hyperoxia-derived lung damage in preterm infants. Semin Fetal Neonatal Med 15: 223-229, 2010.

2. Cordingley JJ and Keogh BF: The pulmonary physician in critical care. 8: Ventilatory management of ALI/ARDS. Thorax 57: 729-734, 2002.

3. Esteban A, Anzueto A, Alia I, Gordo F, Apezteguía C, Pálizas F, Cide D, Goldwaser R, Soto L, Bugedo G, et al: How is mechanical ventilation employed in the intensive care unit? An international utilization review. Am J Respir Crit Care Med 161: 1450-1458, 2000 .

4. Wang H, Liao H, Ochani M, Justiniani M, Lin X, Yang L, Al-Abed Y, Wang H, Metz C, Miller EJ, et al: Cholinergic agonists inhibit HMGB1 release and improve survival in experimental sepsis. Nat Med 10: 1216-1221, 2004.

5. Kallet RH and Matthay MA: Hyperoxic acute lung injury. Respir Care. 58: 123-141, 2013.

6. Sinclair SE, Altemeier WA, Matute-Bello G and Chi EY: Augmented lung injury due to interaction between hyperoxia and mechanical ventilation. Crit Care Med 32: 2496-2501, 2004.

7. Bhandari V and Elias JA: Cytokines in tolerance to hyperoxia-induced injury in the developing and adult lung. Free Radic Biol Med 41: 4-18, 2006.

8. McGrath-Morrow SA and Stahl J: Apoptosis in neonatal murine lung exposed to hyperoxia. Am J Respir Cell Mol Biol 25 $150-155,2001$.

9. Radomski A, Sawicki G, Olson DM and Radomski MW: The role of nitric oxide and metalloproteinases in the pathogenesis of hyperoxia-induced lung injury in newborn rats. Br J Pharmacol 125: 1455-1462, 1998.

10. Klings ES, Lowry MH, Li G, Jean JC, Fernandez BO, Garcia-Saura MF, Feelisch $M$ and Joyce-Brady $M$ : Hyperoxia-induced lung injury in gamma-glutamyl transferase deficiency is associated with alterations in nitrosative and nitrative stress. Am J Pathol 175: 2309-2318, 2009.

11. Allen BW, Demchenko IT and Piantadosi CA: Two faces of nitric oxide: Implications for cellular mechanisms of oxygen toxicity. J Appl Physiol (1985) 106: 662-667, 2009.

12. Bhandari V: Molecular mechanisms of hyperoxia-induced acute lung injury. Front Biosci 13: 6653-6661, 2008.
13. van Zoelen MA, Ishizaka A, Wolthuls EK, Choi G, van der Poll T and Schultz MJ: Pulmonary levels of high-mobility group box 1 during mechanical ventilation and ventilator-associated pneumonia. Shock 29: 441-445, 2008.

14. Sauve AA, Wolberger C, Schramm VL and Boeke JD: The biochemistry of sirtuins. Annu Rev Biochem 75: 435-465, 2006.

15. Guarente L and Picard F: Calorie restriction-the SIR2 connection. Cell 120: 473-482, 2005.

16. Lin SJ, Defossez PA and Guarente L: Requirement of NAD and SIR2 for life-span extension by calorie restriction in Saccharomyces cerevisiae. Science 289: 2126-2128, 2000.

17. Lin SJ, Kaeberlein M, Andalis AA, Sturtz LA, Defossez PA, Culotta VC, Fink GR and Guarente L: Calorie restriction extends Saccharomyces cerevisiae lifespan by increasing respiration. Nature 418: 344-348, 2002.

18. Michan S and Sinclair D: Sirtuins in mammals: Insights into their biological function. Biochem J 404: 1-13, 2007.

19. Taylor DM, Maxwell MM, Luthi-Carter R and Kazantsev AG: Biological and potential therapeutic roles of sirtuin deacetylases. Cell Mol Life Sci 65: 4000-4018, 2008.

20. Shi T, Wang F, Stieren E and Tong Q: SIRT3, a mitochondrial sirtuin deacetylase, regulates mitochondrial function and thermogenesis in brown adipocytes. J Biol Chem 280: 13560-13567, 2005.

21. Park SH, Ozden O, Jiang H, Cha YI, Pennington JD, Aykin-Burns N, Spitz DR, Gius D and Kim HS: Sirt3, mitochondrial ROS, ageing, and carcinogenesis. Int J Mol Sci 12: 6226-6239, 2011

22. Wispé JR, Warner BB, Clark JC, Dey CR, Neuman J, Glasser SW, Crapo JD, Chang LY and Whitsett JA: Human Mn-superoxide dismutase in pulmonary epithelial cells of transgenic mice confers protection from oxygen injury. J Biol Chem 267: 23937-23941, 1992.

23. Slot JW, Geuze HJ, Freeman BA and Crapo JD: Intracellular localization of the copper-zinc and manganese superoxide dismutases in rat liver parenchymal cells. Lab Invest 55: 363-371, 1986.

24. Oury TD, Chang LY, Marklund SL, Day BJ and Crapo JD: Immunocytochemical localization of extracellular superoxide dismutase in human lung. Lab Invest 70: 889-898, 1994.

25. Kingston RE, Chen CA and Rose JK: Calcium phosphate transfection. Curr Protoc Mol Biol 9: 9 1, 2003.

26. Zhang Y, Lin X, Koga K, Takahashi K, Linge HM, Mello A, Laragione T, Gulko PS and Miller EJ: Strain differences in alveolar neutrophil infiltration and macrophage phenotypes in an acute lung inflammation model. Mol Med 17: 780-789, 2011.

27. Mantell LL, Shaffer TH, Horowitz S, Foust R III, Wolfson MR, Cox C, Khullar P, Zakeri Z, Lin L, Kazzaz JA, et al: Distinct patterns of apoptosis in the lung during liquid ventilation compared with gas ventilation. Am J Physiol Lung Cell Mol Physiol 283: L31-L41, 2002.

28. Nishina K, Mikawa K, Takao Y, Maekawa N, Shiga M and Obara H: ONO-5046, an elastase inhibitor, attenuates endotoxin-induced acute lung injury in rabbits. Anesth Analg 84: 1097-1103, 1997.

29. Livak KJ and Schmittgen TD: Analysis of relative gene expression data using real-time quantitative PCR and the 2(-Delta Delta C(T)) method. Methods 25: 402-408, 2001.

30. Numata M, Suzuki S, Miyazawa N, Miyashita A, Nagashima Y, Inoue $S$, Kaneko $T$ and Okubo T: Inhibition of inducible nitric oxide synthase prevents LPS-induced acute lung injury in dogs. J Immunol 160: 3031-3037, 1998.

31. Sun Y, Oberley LW and Li Y: A simple method for clinical assay of superoxide dismutase. Clin Chem 34: 497-500, 1988.

32. North BJ and Verdin E: Sirtuins: Sir2-related NAD-dependent protein deacetylases. Genome Biol 5: 224, 2004.

33. Vassilopoulos A, Fritz KS, Petersen DR and Gius D: The human sirtuin family: Evolutionary divergences and functions. Hum Genomics 5: 485-496, 2011

34. Feldman JL, Dittenhafer-Reed KE and Denu JM: Sirtuin catalysis and regulation. J Biol Chem 287: 42419-42427, 2012.

35. Sauve AA and Youn DY: Sirtuins: NAD(+)-dependent deacetylase mechanism and regulation. Curr Opin Chem Biol 16: 535-543, 2012.

36. Hallows WC, Albaugh BN and Denu JM: Where in the cell is SIRT3?-functional localization of an NAD+-dependent protein deacetylase. Biochem J 411: e11-e13, 2008.

37. Buler M, Aatsinki SM, Izzi V and Hakkola J: Metformin reduces hepatic expression of SIRT3, the mitochondrial deacetylase controlling energy metabolism. PLoS One 7: e49863, 2012. 
38. Hallows WC, Lee S and Denu JM: Sirtuins deacetylate and activate mammalian acetyl-CoA synthetases. Proc Natl Acad Sci USA 103: 10230-10235, 2006.

39. Davis JM, Rosenfeld WN, Richter SE, Parad MR, Gewolb IH, Spitzer AR, Carlo WA, Couser RJ, Price A, Flaster E, et al: Safety and pharmacokinetics of multiple doses of recombinant human $\mathrm{CuZn}$ superoxide dismutase administered intratracheally to premature neonates with respiratory distress syndrome. Pediatrics 100: 24-30, 1997.

40. Nakamura T and Ogawa Y: Prophylactic effects of recombinant human superoxide dismutase in neonatal lung injury induced by the intratracheal instillation of endotoxin in piglets. Biol Neonate 80: 163-168, 2001.

41. Jacobson JM, Michael JR and Jafri MH Jr and Gurtner GH: Antioxidants and antioxidant enzymes protect against pulmonary oxygen toxicity in the rabbit. J Appl Physiol (1985) 68 1252-1259, 1990 .

42. Tanswell AK, Olson DM and Freeman BA: Liposome-mediated augmentation of antioxidant defenses in fetal rat pneumocytes. Am J Physiol 258: L165-L172, 1990.

43. Walther FJ, Gidding CE, Kuipers IM, Willebrand D, Bevers EM, Abuchowski A and Viau AT: Prevention of oxygen toxicity with superoxide dismutase and catalase in premature lambs. J Free Radic Biol Med 2: 289-293, 1986.

44. Freeman BA, Topolosky MK and Crapo JD: Hyperoxia increases oxygen radical production in rat lung homogenates. Arch Biochem Biophys 216: 477-484, 1982.

45. Fox RB, Hoidal JR, Brown DM and Repine JE: Pulmonary inflammation due to oxygen toxicity: Involvement of chemotactic factors and polymorphonuclear leukocytes. Am Rev Respir Dis 123: 521-523, 1981 .

46. Mantell LL and Lee PJ: Signal transduction pathways in hyperoxia-induced lung cell death. Mol Genet Metab 71: 359-370, 2000.

47. Jamieson D: Oxygen toxicity and reactive oxygen metabolites in mammals. Free Radic Biol Med 7: 87-108, 1989.

48. Ansari A, Rahman MS, Saha SK, Saikot FK, Deep A and Kim KH: Function of the SIRT3 mitochondrial deacetylase in cellular physiology, cancer, and neurodegenerative disease. Aging Cell 16: 4-16, 2017.

49. Tao R, Coleman MC, Pennington JD, Ozden O, Park SH, Jiang H, Kim HS, Flynn CR, Hill S, Hayes McDonald W, et al: Sirt3-mediated deacetylation of evolutionarily conserved lysine 122 regulates MnSOD activity in response to stress. Mol Cell 40: 893-904, 2010
50. Candas D and Li JJ: MnSOD in oxidative stress response-potential regulation via mitochondrial protein influx. Antioxid Redox Signal 20: 1599-1617, 2014.

51. Bause AS, Matsui MS and Haigis MC: The protein deacetylase SIRT3 prevents oxidative stress-induced keratinocyte differentiation. J Biol Chem 288: 36484-36491, 2013.

52. Rahman I and MacNee W: Lung glutathione and oxidative stress: Implications in cigarette smoke-induced airway disease. Am J Physiol 277: L1067-L1088, 1999.

53. Bargagli E, Olivieri C, Bennett D, Prasse A, Muller-Quernheim J and Rottoli P: Oxidative stress in the pathogenesis of diffuse lung diseases: A review. Respir Med 103: 1245-1256, 2009.

54. Mach WJ, Thimmesch AR, Pierce JT and Pierce JD Consequences of hyperoxia and the toxicity of oxygen in the lung. Nurs Res Pract 2011: 260482, 2011.

55. Borok Z, Buhl R, Grimes GJ, Bokser AD, Hubbard RC, Holroyd KJ, Roum JH, Czerski DB, Cantin AM and Crystal RG: Effect of glutathione aerosol on oxidant-antioxidant imbalance in idiopathic pulmonary fibrosis. Lancet 338: 215-216, 1991.

56. Kinnula VL, Fattman CL, Tan RJ and Oury TD: Oxidative stress in pulmonary fibrosis: A possible role for redox modulatory therapy. Am J Respir Crit Care Med 172: 417-422, 2005.

57. Johnson LN and Koval M: Cross-talk between pulmonary injury, oxidant stress, and gap junctional communication. Antioxid Redox Signal 11: 355-367, 2009.

58. Vivekananda J, Lin A, Coalson JJ and King RJ: Acute inflammatory injury in the lung precipitated by oxidant stress induces fibroblasts to synthesize and release transforming growth factor-alpha. J Biol Chem 269: 25057-25061, 1994.

59. Guo X, Kesimer M, Tolun G, Zheng X, Xu Q, Lu J, Sheehan JK, Griffith JD and Li X: The NAD(+)-dependent protein deacetylase activity of SIRT1 is regulated by its oligomeric status. Sci Rep 2: 640, 2012.

60. Sundaresan NR, Gupta M, Kim G, Rajamohan SB, Isbatan A and Gupta MP: Sirt3 blocks the cardiac hypertrophic response by augmenting Foxo3a-dependent antioxidant defense mechanisms in mice. J Clin Invest 119: 2758-2771, 2009.

61. Zhang B, Cui S, Bai X, Zhuo L, Sun X, Hong Q, Fu B, Wang J, Chen X and Cai G: SIRT3 overexpression antagonizes high glucose accelerated cellular senescence in human diploid fibroblasts via the SIRT3-FOXO1 signaling pathway. Age (Dordr) 35: 2237-2253, 2013. 